\title{
SISTEMA COLABORATIVO DE REGISTRO E ORGANIZAÇÃO DE DADOS SOBRE A HISTÓRIA DE CONSTITUIÇÃO DE UM SABER, APLICADO AO CASO DA MODELAGEM GEOMÉTRICA.
}

\section{COLLABORATIVE SYSTEM FOR REGISTRATION AND ORGANIZATION OF CONTENTS ON THE HISTORICAL CONSTITUTION OF A KNOWLEDGE, APPLIED TO THE CASE OF GEOMETRIC MODELING}

\author{
Vinicius Batista Fagundes ${ }^{1}$ \\ Adriane Borda Almeida da Silva² \\ Neusa Mariza Rodrigues Félix ${ }^{3}$
}

\section{RESUMO}

O presente trabalho aborda o desenvolvimento de uma ferramenta para a web, disponível através da internet, que permita o estabelecimento de uma atividade colaborativa de compilação, análise, discussão e sistematização de dados sobre a constituição de um determinado saber, ou de uma disciplina. Tal ferramenta tem a finalidade de permitir, além desse processo de construção colaborativa, a consulta aberta sobre os dados, para apoiar atividades científicas e didáticas. O experimento está sendo realizado aplicando-se ao caso da história da constituição dos saberes relativos à Modelagem Geométrica, em princípio, como apoio às atividades científicas e didáticas na área de representação gráfica. Neste momento a ferramenta encontra-se no estágio de experimentação no âmbito do grupo de pesquisa.

Palavras chave: sistema colaborativo, dados históricos, modelagem geométrica.

\section{ABSTRACT}

The present work approaches the development of a tool for web, available through the Internet, capable to promote a collaborative construction, analysis, and systematization of information related to the constitution of an specific area of knowledge. The tool is built to permit the collaborative work and also the remote access, aiming to support didactic and scientific activities. The experiment is being developed to the case of historical constitution of geometric modeling, firtly as a support to the activities related to graphic representation. At the present stage the tool is being tested by the research group.

Words key: Historical Colaborativo System, Data, Geometric Modeling

\section{Introdução}

A presença da informática vem sendo cada vez mais requerida, nos diferentes setores da sociedade e nas diversas áreas do conhecimento, na tentativa de fomentar e dinamizar os processos produtivos.

Considera-se que atividades ainda não permeadas pelas tecnologias da informação e da comunicação deixam de usufruir a possibilidade de implementar um ritmo de

\footnotetext{
${ }^{1}$ Funcionário, Graduação em Analise de Sistemas, função de Analista de Tecnologia da Informação na Área de EAD/UFPel. vbfagundes@gmail.com

${ }^{2}$ Professora Adjunta Doutora do Departamento de Desenho Técnico e Gráfica Computacional, IFM/UFPel, GEGRADI, adribord@ufpel.tche.br

3 Professora Titular Doutora do Departamento de Desenho Técnico e Gráfica Computacional, IFM/UFPel, GEGRADI, neusarf@ufpel.tche.br

V. $4 \mathrm{~N}^{\circ} 1$, Julho, 2006
} 
desenvolvimento mais acelerado e otimizado, permitido pelos processos de automatização e de conexões em rede. Quando exploradas, estas tecnologias têm promovido novas formas de relacionamento pessoal e profissional, em diferentes setores da sociedade e áreas do conhecimento.

Particularmente, no setor educativo, o uso destas tecnologias como mediadoras nos processos de ensino/aprendizagem têm provocado reflexões importantes e estabelecido uma área específica de estudo, a EAD (Educação a Distância). O presente trabalho situa-se nesta área de interesse. É desenvolvido pelo Grupo GEGRADI, Grupo de Estudos para o Ensino/aprendizagem de Gráfica Digital.

A questão estudada parte da necessidade de sistematizar dados históricos capazes de fundamentar o ensino e a pesquisa em Gráfica Digital. Esta área se estabelece a partir da inserção da tecnologia informática como potencializadora da atividade de representação gráfica. Faz-se necessário explicitar os saberes de referência adotada pela Gráfica Digital, suas origens e processos de constituição. O reconhecimento desta história promove a revisão e ampliação dos saberes que atualmente delimitam a área de representação gráfica.

Porém, esta atividade é complexa e quase ilimitada, tendo em vista as constantes conexões que são criadas entre novos saberes e àqueles já sistematizados, propondo outras reorganizações. Deve-se considerar que a cada leitura de um mesmo dado, sob outra perspectiva teórica ou tecnológica, é possível atribuir novos significados a estes mesmos dados, incrementando a história abordada.

Este tipo de trabalho vem-se desenvolvendo no âmbito do Curso de pós-graduação em Gráfica Digital, buscando-se a partir das possibilidades oferecidas pelas tecnologias de informação e comunicação, contar com a colaboração de outros pesquisadores, de diferentes áreas do conhecimento.

Desta forma, tem-se o propósito de gerar um sistema que permita a compilação, análise, discussão e sistematização de dados sobre a constituição dos saberes relativos à Modelagem Geométrica. Onde, via internet, se estabeleça um processo de construção colaborativa e que ainda permita a consulta aberta sobre os dados, para apoiar atividades científicas e didáticas.

\section{A Motivação e o Sistema Proposto}

Este estudo parte do propósito de compreensão da dimensão genética de um saber, que faz parte da metodologia de trabalho apontada pela Teoria Antropológica da Didática (CHEVALLARD, 1991), e que constitui uma linha de pesquisa já delimitada em Borda, 2001. A Teoria Antropológica da Didática amplia a noção de saber ao conceito de praxeologia. Este conceito refere-se à identificação de uma estrutura formal do saber, composta por quatro elementos que se relacionam de forma dinâmica e dialética: tarefas, técnicas, tecnologias e teorias. Yves Chevallard considera que cada um destes elementos adquire características próprias, de acordo com o contexto em que se insere. $\mathrm{O}$ saber sofre transformações quando transita desde o contexto em que é produzido, e utilizado, até o contexto em que é reconhecido, sistematizado e veiculado, como em um contexto de ensino. $\mathrm{O}$ estudo deste fenômeno de trânsito do saber é do que se ocupa a Teoria da Transposição Didática, que se insere então na Teoria Antropológica da Didática. Identificar a dimensão genética do saber significa, à luz destas teorias, 
observar as reorganizações que ao longo do tempo vão ocorrendo para constituir um saber em um contexto específico (CHEVALLARD,1991). Faz-se necessário, então, partir da análise de dados que descrevam a história deste processo, para identificar os diversos elementos que vão compondo o saber estudado, em cada estágio de desenvolvimento teórico e tecnológico.

A partir deste marco teórico, foram desenvolvidas práticas didáticas, com propostas de trabalho colaborativo entre alunos e professores, com o objetivo de estruturar materiais didáticos que facilitassem o estudo, descritas em BORDA (2005). Estas práticas resultaram em uma quantidade significativa de imagens e informações textuais, relativas ao tema tratado, exigindo a criação de um sistema capaz de armazenar e manipular os dados referidos.

A principal característica buscada para o sistema é a facilidade de uso, associada ao acesso e manipulação on-line. Em termos de informações, deve permitir o cadastro dos usuários e dos conteúdos a serem armazenados no Banco de Dados.

O formato em que se encontrava este material não permitia o estabelecimento de um processo colaborativo na modalidade à distância. Passou-se, então, a explorar as facilidades oferecidas pelas tecnologias de informação e comunicação, via internet. Para isso, constituiu-se uma equipe interdisciplinar capaz de estruturar um sistema informático de banco de dados que permita organizar e inter-relacionar este acervo de informações de acordo com interesses selecionados pelo próprio pesquisador.

Estuda-se um desenho da interface do ambiente adequado ao perfil dos possíveis usuários do sistema. Estes usuários estão atualmente caracterizados por pesquisadores, professores e estudantes relacionados com a Informática Gráfica e as aplicações deste campo nas diferentes áreas do conhecimento, incluindo, principalmente, àqueles que buscam a compreensão do processo de potencialização da atividade de representação da geometria a partir da apropriação da tecnologia informática nesta atividade.

Deve-se destacar que os usuários terão um papel importante para futuras implementações, participando com sugestões e críticas no processo contínuo de aprimoramento do sistema.

\section{Processo de Desenvolvimento do Sistema}

O Sistema idealizado surge, então, com a finalidade de disponibilizar um ambiente colaborativo para a construção de uma base de dados sobre a História da Modelagem Geométrica, que permita uma consulta aberta, para apoiar atividades didáticas e estimular a pesquisa sobre o tema tratado. $\mathrm{O}$ sistema tem sido projetado para atender aos requisitos delimitados na seção anterior, observando-se que ao longo de sua utilização será possível a identificação de outros requisitos para os quais o sistema terá que ser adaptado.

Segundo BECK (2000), um dos principais problemas relacionados ao desenvolvimento de software é o fator de risco. A mudança de requisitos durante o processo de desenvolvimento é um risco permanente. Tal mudança poderá exigir reformulações profundas no sistema, por vezes, ocasionando a inviabilidade do projeto inicial e a necessidade de elaboração de um projeto de software totalmente novo. 
Para minimizar as conseqüências destas mudanças, segue-se a orientação de BECK (2000). Adota-se algumas práticas da metodologia XP, Extreme Programming. Segundo este autor, é uma metodologia de desenvolvimento de software a qual assume que os requisitos do sistema mudam constantemente. É considerada uma metodologia ágil e está baseada nos valores: simplicidade, comunicação e feedback, além do valor coragem, que se refere à iniciativa de mudanças no momento exato de solicitação.

\subsection{Planejamento}

O sistema foi planejado de acordo com os valores sugeridos por BECK (2000).

O valor simplicidade passa a ser atendido por conseqüência da adoção da metodologia $\mathrm{XP}$, a qual considera que o próprio código é o Design do sistema. Com o objetivo de melhorar o Design simplifica o código, removendo linhas de código duplicadas e aumentando a coesão. Em XP leva-se isto a níveis extremos, sem permitir que se implemente funções adicionais que não serão usadas na atual iteração. Desta forma, o projeto deve começar simples e se manter simples, através de testes e refinamento do design.

O valor comunicação, em um primeiro momento, se estabelece em duas instâncias: 1) no planejamento de uma versão do sistema, onde o usuário propõe funcionalidades desejadas e o Desenvolvedor avalia a dificuldade de implementá-las. 2) no planejamento das iterações, onde o usuário define as funcionalidades prioritárias para a iteração e o Desenvolvedor as subdivide em tarefas.

As tarefas foram subdivididas, nesta primeira versão do sistema, da seguinte forma:

- Criação de Base de dados - Local onde será armazenada toda a informação cadastrada no sistema, tanto dados pessoais dos usuários como Informações referentes à História da Modelagem Geométrica;

- Login - módulo de acesso ao sistema, através de uma senha.

- Cadastro de usuários - módulo do sistema que registra os dados pessoais dos usuários no Banco de Dados, um dos requisitos para a emissão de senha;

- Incluir Evento Histórico - é o módulo mais complexo do sistema, que permite a inserção das Informações referentes à História da Modelagem Geométrica no Banco de Dados;

- Pesquisas - módulo que permite a busca de informações específicas, cadastradas no Banco de dados;

- Mural - módulo para a interação entre os usuários (colaboradores), postando sugestões e comentários sobre as informações armazenadas no sistema.

- Painel Administrativo - permite o acesso a todo o sistema, restrito aos administradores. Através deste painel o administrador terá controle ás informações dos usuários e aos conteúdos armazenados por eles. 
O valor feedback refere-se ao processo de construção do sistema, sempre a partir de pequenos lançamentos, para que possa ser testado e avaliado sobre cada intervenção específica. Por exemplo, pode-se começar pela implementação de um único módulo ou até mesmo, parte de um módulo. De acordo com o sucesso da implementação segue-se adicionando cada uma das outras partes do sistema. Adota-se esta prática durante todo o processo de desenvolvimento, garantindo que a cada iteração o software funcione plenamente. Isto permite que problemas sejam detectados em processos iniciais, a partir da comunicação entre o usuário e o desenvolvedor, em cada uma das fases de implementação do sistema.

\section{Linguagens Utilizadas}

Partiu-se da definição prévia de que o sistema deveria ser desenvolvido para WEB e ainda permitir o acesso remoto a uma Base de Dados. Desta forma, e após a etapa de Planejamento, buscou-se definir os tipos de Linguagens adequadas para o Desenvolvimento do Sistema.

Para a manipulação de dados adota-se o MySQL que, segundo SUEHRING (2002), é um Sistema de Gerenciamento de Banco de Dados, SGBD, com uma estrutura regular que organizam informações e que utiliza a linguagem SQL (Structured Query Language - Linguagem de Consulta Estruturada) como interface.

As principais características do MySQL são:

- Portabilidade, por suportar praticamente qualquer plataforma atual;

- Excelente desempenho e estabilidade;

- Pouco exigente em termos de recursos de hardware;

- Facilidade de uso;

- É um Software Livre;

- Fácil integração com o PHP (Hypertext Preprocessor).

Considerando-se esta última característica de um Sistema MySQL e tendo-se a necessidade de adotar uma linguagem de programação voltada para web, utilizou-se o PHP, que é uma linguagem, de código aberto, apropriada para a criação de aplicativos em ambiente de rede. Os scripts PHP são executados no servidor web para a manipulação de páginas.

Para a estruturação destas páginas web adota-se a linguagem HTML. Esta linguagem, basicamente, trata de um conjunto de etiquetas que servem para definir a forma na qual se apresentará o texto e outros elementos da página.

\section{Desenvolvimento do Sistema}

Seguindo-se o Planejamento, apoiando-se na metodologia XP, a primeira atividade de implementação do sistema foi à criação da Base de Dados. A Figura 1 mostra o Banco de Dados que foi desenvolvido utilizando a ferramenta phpMyAdmin, que proporciona a interface para o desenvolvedor criar e controlar as informações na Base de Dados.

Assim, utilizando a Linguagem SQL foram criadas várias tabelas: de conteúdo (para armazenar informações referentes à modelagem geométrica, inseridas no módulo Cadastro de conteúdos); de dados (para armazenar as informações pessoais dos usuários, inseridas no módulo Cadastro de Usuários); mensagens (para armazenar as 
informações inseridas no módulo Contato); thumb_img (para armazenar as informações inseridas no módulo Cadastro de Símbolos).

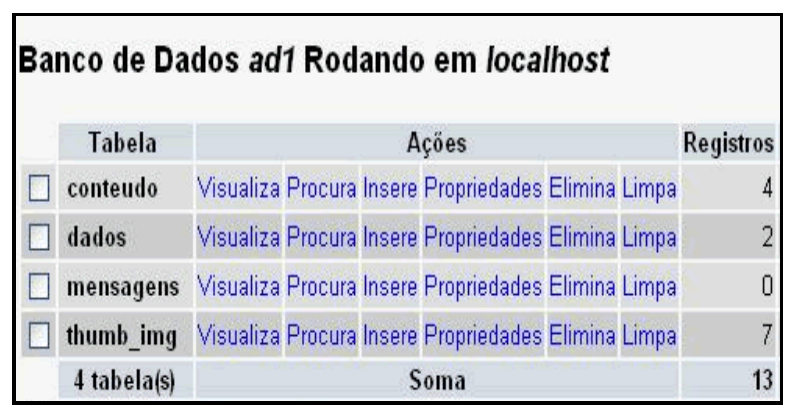

Figura 1: Banco de Dados

Fonte: do autor.

Baseado nos campos criados nestas tabelas da Figura 1, passou-se a implementar a interface inicial do sistema com a definição das interfaces dos distintos módulos.

Iniciou-se com os campos usuário e senha da tabela dados, considerando-se duas situações padrão: a dos usuários já cadastrados e que estão liberados para o acesso através de senha individual; a dos usuários que ainda necessitam ser cadastrados para a posterior liberação de senha de acesso. Neste segundo caso, o sistema direciona o solicitante para uma nova interface que possui um formulário de cadastro.

Depois de realizado o acesso, o usuário estará apto a navegar pelo sistema e terá a sua disposição um menu principal de navegação para os Módulos: Conteúdo, Pesquisa e Contato.

No Módulo Incluir Evento Histórico, mostrado na Figura 2, foram criados campos a serem preenchidos que se referem a informações sobre o tipo de conteúdo inserido (metadados), para posteriormente facilitar o acesso direcionado ou seletivo a estas informações.

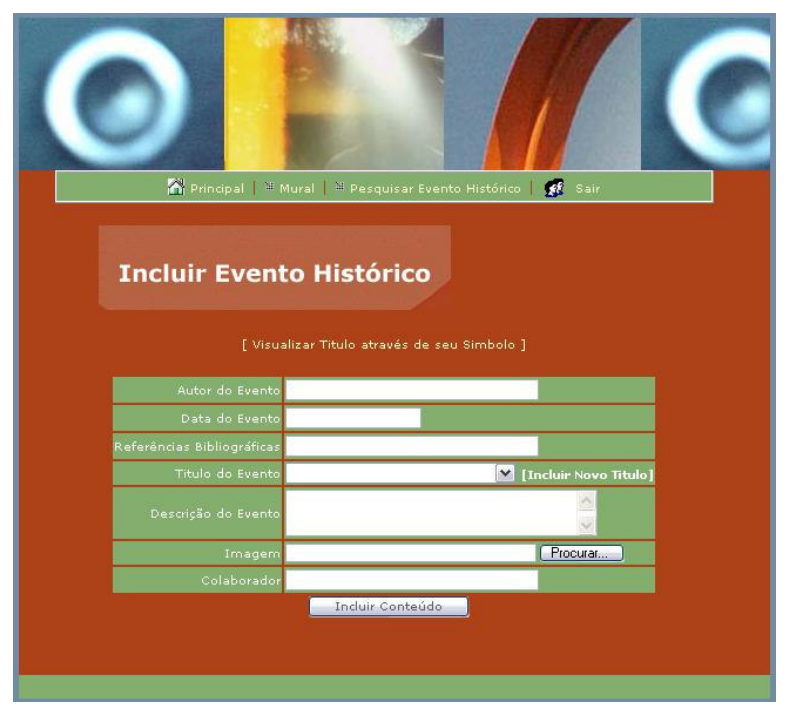

Figura 2: Incluir Evento Histórico

Fonte: do autor. 
O campo relativo à seleção de símbolos, que devem ser associados a cada informação adicionada, refere-se à categorização do próprio conteúdo dentro da área de conhecimento. Os dados já devem ser categorizados no momento da sua inserção no sistema. Desta forma são disponibilizados símbolos, com seus respectivos significados, para que o responsável pela introdução destes dados já realize uma sugestão sobre qual ou quais deles deverão ser associados aos dados referidos. No trabalho inicial, em BORDA (2001), foi adotada uma simbologia para representar cada área, exemplificada na Legenda de Símbolos na Figura 3.

Os símbolos disponibilizados neste campo, provisoriamente, são àqueles adotados em BORDA (2001). Segue-se a proposta de relacionar eventos científicos com a conformação de áreas da geometria, como por exemplo, as análises realizadas em RODRIGUEZ-SANTURJO (1998), sobre a delimitação da Geometria Projetiva. Além dos dados referentes ao desenvolvimento dos saberes propriamente ditos, buscam-se dados necessários para contextualizar a infra-estrutura tecnológica existente em cada momento histórico, definindo a categoria denominada suporte físico.

$\begin{array}{ll}\text { Titulo } & \text { Simbolo } \\ \text { - Soporte Físico } & \\ \text { - Curvas } & 5 \\ \text { - Geometría Analítica } & 1\end{array}$

Figura 3: Legenda de Símbolos

Fonte: do autor.

No Módulo Pesquisas, mostrado na Figura 4, os usuários terão à sua disposição várias possibilidades de realizar buscas de informações específicas na Base de Dados.

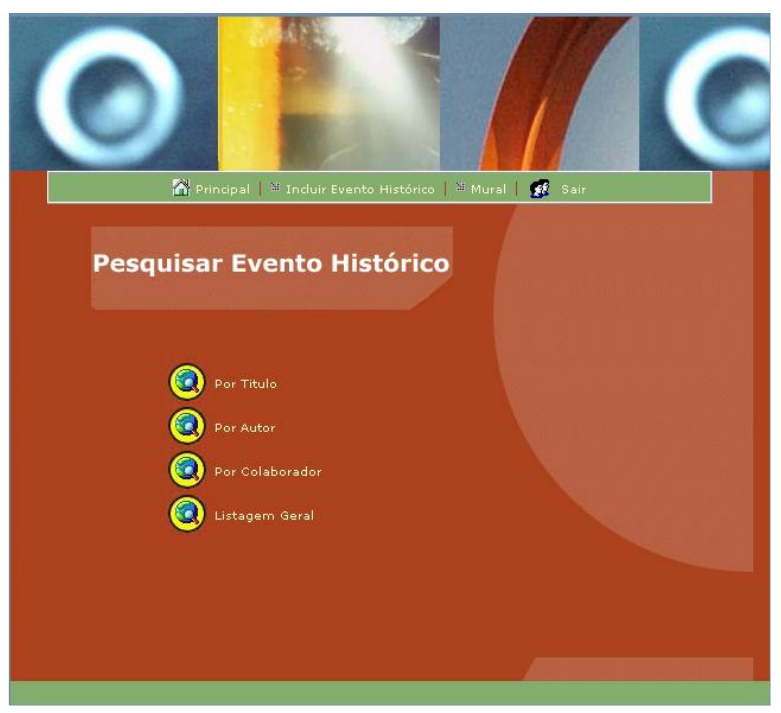

Figura 4: Pesquisas

Fonte: do autor

No Módulo Mural o colaborador pode deixar mensagens que pode ser visualizada por todos os usuários, aumentado assim o poder de interação do sistema. 
Para o acesso às tarefas de gerenciamento do sistema, existe logicamente, uma senha restrita ao administrador, utilizando-se, para este acesso, este módulo possui uma interface diferente da que é utilizada pelos demais usuários.

\section{Conclusões e Perspectivas}

Avalia-se, neste momento, que a utilização dos conceitos relativos à metodologia XP proporcionou organizar, de modo ágil, o processo de desenvolvimento do sistema. Além disso, considera-se que as tecnologias empregadas como MYSQL e o próprio PHP, demonstraram-se compatíveis com esta metodologia utilizada.

Os testes realizados pelo próprio grupo de desenvolvimento permitem vislumbrar a possibilidade de que neste ambiente colaborativo se estabeleça um processo contínuo de revisão e sistematização da História da Modelagem Geométrica, de significativa contribuição ao contexto que se insere este trabalho.

Pretende-se que este projeto assuma, desta forma, um caráter permanente de desenvolvimento, por não existir um limite tanto para a tarefa de busca de dados como para o aperfeiçoamento de um sistema de manipulação de dados.

Neste momento, uma das principais metas definida para a continuidade do projeto é a implementação da possibilidade do trabalho colaborativo ser desenvolvido de forma síncrona.

Avaliam-se também propostas de adequações deste mesmo sistema para o estudo de outras disciplinas, tanto de interesse específico do Grupo GEGRADI e do Curso de Especialização em Gráfica Digital, como de outras áreas do conhecimento.

Considera-se que intensificar o uso deste sistema garante o estabelecimento de um processo de aperfeiçoamento dos materiais e métodos empregados.

\section{Referências Bibliográficas}

BORDA, A. Lucas,A., Felix,N. 2005 Processo Colaborativo de Leitura da História da Modelagem Geométrica. GRAPHICA 2005 Recife Brasil

CHEVALLARD, Yves. "La Transposition Didactique. Du savoir savant au savoir enseigné”. La Pensée Sauvage, 2ème édition. Grenoble, 1991.

BORDA, Adriane. Los saberes constitutivos del Modelado Geométrico y Visual, desde las instituciones científicas y profesionales a las escuelas de arquitectura. Tese de Doutorado, Universidad de Zaragoza, Zaragoza, Espanha, Setembro, 2001.

RODRÍGUEZ-SANJURJO, J.M; RUIZ, J.M. Geometría Proyectiva. AddisonWesley, 1998.

BECK, Kent. Extreme Programming Explained. Addison-Wesley, 2000.

SUEHRING, Steve. MySQL - A Biblia. Campus, 2002 